\title{
Design of the CNC System for Vertical Electrochemical Machining Tool Based on Siemens 802Ds1
}

\author{
Siguo Yang \\ Chuzhou Vocational and Technical College, Chuzhou City (Post Code: 239000),Anhui Province, China
}

\begin{abstract}
The common MCU (Microcontroller Unit) or PLC control method can not meet the linkage processing. 802Dsl numerical control system is adopted in this paper so that four axis and above linkage machining control can be realized in vertical ECM tool which has the advantages of complete functions, high reliability and good stability. With the support of my tutor - Professor / Dr. Chen Yuanlong and his graduate team, design, installation and debugging of $\mathrm{NC}$ system have been accomplished according to the modular design method of software side. It has been successfully applied in special processing laboratory of $\mathrm{HeFei}$ University of Technology. The design system has a wide range of needs for small and medium enterprises requiring complex molding surface and cavity processing.
\end{abstract}

Keywords-electrochemical machining tool; 802Dsl; CNC system; Linkage

\section{INTRODUCTION}

EMC adopts the principle that the metal produces anodic dissolution in the electrolyte to achieve the processing of the metal parts, which is mainly used for the machining of the difficult-to-machine material, complicated molded surface and cavity. EMC has great significance in the mechanical manufacturing industry[1]. With the rapid development of science and technology, the parts'structure has become increasingly complicated, which has a high requirements for the machining accuracy and control ability, but the common MCU or PLC control method can't meet the linkage machining. This thesis adopts 802Dsl system to design 4-axis linkage ECM machine tool control system for solving the problem.

\section{A. The Structure of DJL3163 Machine Tool}

As machine tool adopts the structure of four column type, it has a strong rigidity and good reliability. The machine tool is equipped with $\mathrm{X} / \mathrm{Y} / \mathrm{Z}$ three feeding axis and a rotation axis $\mathrm{A}$, and each axis is driven by AC servo motor. The feed axis motor drives the working platform to work by the ball screw drive mechanism, and the mechanical stop unit and the hard limit switch are installed in the end of the feed axis. As the A axis motor is installed on the rotating workbench equipped with pneumatic locking device, you can use the manual function or $\mathrm{M}$ function to control the disengagement or lock of the axis. Generally, the $\mathrm{X} / \mathrm{Y}$ axis is used for adjusting the cathode position, as to the complicated molded surface, it can be linked with other axis. $\mathrm{Z}$ axis is the main feed axis, which has a high request for the repeated positioning accuracy and feeding accuracy. A axis is used for adjusting the rotary indexing of the workpiece. Figure I shows the structure diagram of machine tool.

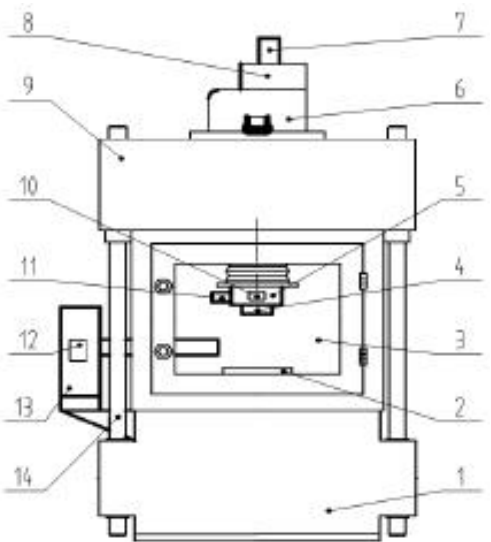

1-Machine body; 2-Workbench; 3-Work box; 4-Cathode installation plates;5-X/Y platform; 6-Lid; 7-Z axis motor;8-Reduction gearbox; 9-Crossbeam; 10-Y axis motor; 11-X axis motor;12-A axis motor;13-Rotating work platform;14-Column

FIGURE I. THE STRUCTURE DIAGRAM OF MACHINE TOOL

\section{B The Design of Hardware System}

Figure II shows the structure of hardware system. The hardware system adopts Siemens 802Dsl CNC system, its main composed units include PCU (Panel Control Unit, Panel Control Unit), NC keyboard, I/O module PP72/48, feed unit(servo motor driving module and power supply module).

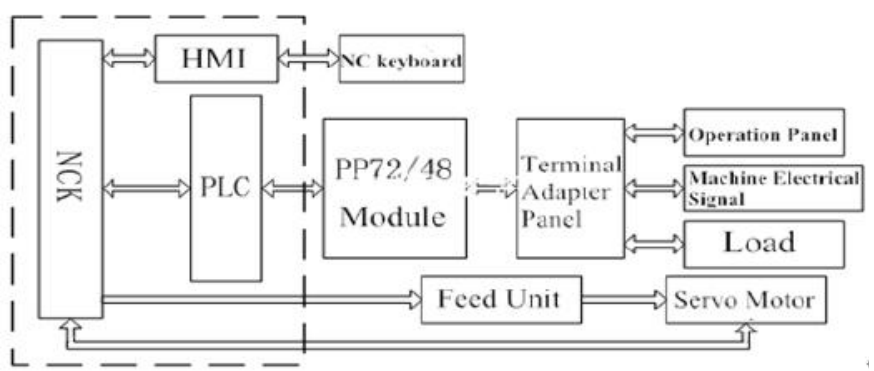

FIGURE II. THE SYSTEM HARDWARE STRUCTURE CHART 
The core part PCU integrates PLC (Programmable Logic Controller), HMI (Human-machine Interface), NCK (Numerical Control Core) together, then it is made into installation plate, and it can drive at most 5 axis and a PLC axis through DRIVE-Cliq interface. The external input and output signal(operation panel control signal and machine electric signal) are connected to I/O module PP72/48 via terminal adapter panel, and the I/O module is connected to internal PLC via ProfiBus,by which can complete machine logic control. This system at most can configure 3 I/O module [2].

The servo driving control of each axis is completed by NCK, and the Servo motor is equipped with position encoder. The closed loop control is implemented by the signal cable which feedbacks the actual rotational speed and position. After eliminating interference via the reactor and low-pass filter power, $380 \mathrm{~V}$ AC becomes $600 \mathrm{~V} \mathrm{DC}$, and then supplied to motor module via the DC bus. The DC motor module turns the $\mathrm{DC}$ into controllable AC to drive the servo motor.

\section{The Designs of Software System}

The Requirements of Control System. (1) The control system of machine tool should be able to receive and respond to the signals quickly and accurately without any interference among all the functions;(2)To avoid the mistakes caused by misoperation, the interlock protection should be set to the signal-input procedure when designing the program and control circuit;(3) The requirements of the electrochemical machining function should be well met, which includes manual/automatic processing function, manual/automatic tool setting function, indervoltage protection and short-circuit protection function, interlocking protection function and so on[3 ].

The Design Plan of Control System. According to the control requirements of machine tool, the control system adopts the modular designs, including tool setting module, preparation module (electrolysis power and cell liquor pump control module), the axis-controlling module, protection module, monitoring module, emergency-stopping module [4]. The software Structures of CNC system are shown in Figure III

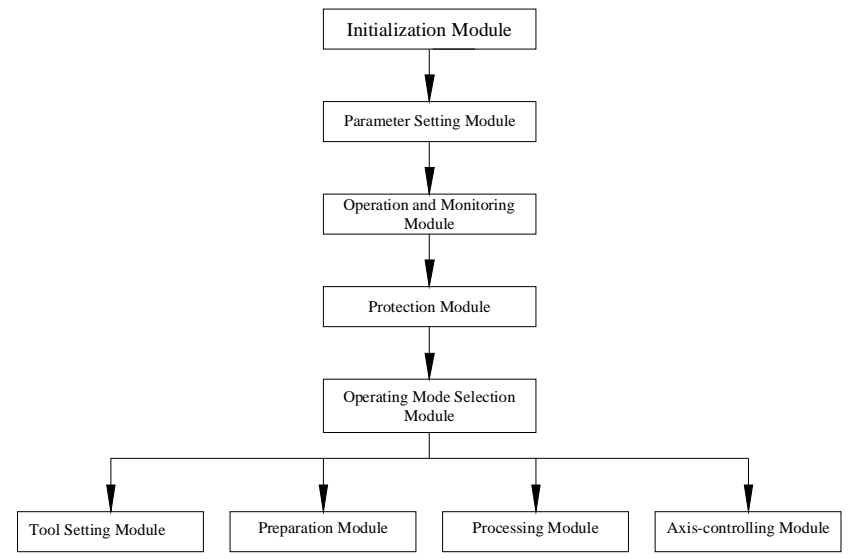

FIGURE III. . .THE STRUCTURE DIAGRAM OF CNC SOFTWARE

(1)In the PLC first scanning cycle, the initialization module invokes the initial program, which is used in the register to set an initial value, to complete the initialization of PLC
(2)The parameter setting module contains generic parameters and process parameters. The common parameter settings mainly include setting jog speed, quicker movement, the tool-setting speed. The processing parameters set different alarm value for different workpiece's requirements.

(3)The monitoring module includes the monitoring of machine operation status and the monitoring of electrochemical machining analog. The monitoring of machine operation condition includes the monitoring of the input and output unit I/O conditions and the monitoring of axes-operating state (speed control, contour control, a hard limit monitoring). The analog monitoring focuses on the electrolyte temperature, flow, pressure, the electrolysis voltage and the electric current and so on, and transforms the analog signals into recorder-connected (4 $\sim 24 \mathrm{~mA})$. According to the range of limit value, the recorder can get the actual value based on the detected current analog signal. The logger can display and store every change of the parameters and alarms. The alarm signal is outputted by the relay.

(4)The protection module includes PLC user alarm, emergency-stopping procedures and interlocking protection program. PLC user alarm system is one of the most effective diagnostic means of protection. PLC detects I/O status information through the PLC user alarm. The alarming responses include PLC stop alarm response, emergency-stop response, supply-keeping, inserting-banning, starting-banning and"display only"alarm Response. The emergency program mainly provides undervoltage protection, overcurrent protection, a hard limit alarm and drive failure protection. The program of interlocking protection is written based on the characteristics of the electrolytic process, in order to avoid damage caused by misoperation, such as the emergence of a tool setting signal power, electrolysis power and the electrolytic pump solution. The pump electrolyte electrolysis power supply cannot turn on with the electrolytic pump unopened.

(5) The selection module of working mode includes the designs of an automatic mode, manual mode, MDA mode (manual programming, automatic execution) and back-to-zero mode design (Axis returns a reference point ).

(6)The tool setting module functions include the tool setting and simulation of the tool setting, the circuit of tool setting is shown in Figure IV.

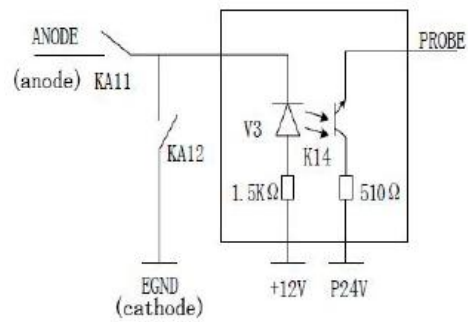

FIGURE IV. SCHEMATIC DIAGRAM SETTING CIRCUIT

In manual mode, after pressing the button on the tool setting, when the cathode and anode contacting with each other caused short-circuit the relay KA11 contact closed. The 
contacts PROBE feedbacks the tool setting signal to the system. When the system is in debugging, the button is pressed for simulating the tool setting system, and the relay KA12 contact closed.

(7) The processing module provides manual processing and automatic processing functions. The manual processing can adjust feed ratio or select the pulse equivalent in the increment of feed.

The Design Of PLC Programming. Programming is carried out with the help of the specialized programming software programming tool PLC 802D 3.1V. The program structure consists of a main program and associated subprograms, using LAD (Ladder) as a programming language. Firstly, based on the control system of the program, we are about the design of the functional modules. The program of the axis control, electrolytic power and electrolyte pump control, driver control, alarming information and handle operations are made into the different functional modules. And then based on the relationship between the various functions, the interlock protection procedure is programmed.

TABLE I. INTERNAL SIGNAL TRANSMISSION ADDRESS TABLE

\begin{tabular}{|c|c|c|c|}
\hline$\underset{r}{\text { Numbe }}$ & $\begin{array}{c}\text { Address range } \\
\text { of variable }\end{array}$ & $\begin{array}{c}\text { Transmission } \\
\text { direction of } \\
\text { Signal }\end{array}$ & Transfer the main contents \\
\hline 1 & $\begin{array}{l}\text { V10000000 } \\
\text { V10000008 }\end{array}$ & $\mathrm{MCP} \rightarrow \mathrm{PLC}$ & $\begin{array}{l}\text { To send the key signal from } \\
\text { MCP to PLC with the data form, } \\
\text { including NC control keys, each } \\
\text { axis-operating control keys, } \\
\text { override-option switch, } \\
\text { users-option keys }\end{array}$ \\
\hline 2 & $\begin{array}{l}\text { V11000000 } \\
\text { V11000007 }\end{array}$ & $\mathrm{PLC} \rightarrow \mathrm{MCP}$ & $\begin{array}{l}\text { To send the PLC back to the } \\
\text { P MCP (except for the } \\
\text { override-option switch) }\end{array}$ \\
\hline 3 & $\begin{array}{l}\text { V16000000 } \\
\text { V16000007 }\end{array}$ & $\mathrm{PLC} \rightarrow \mathrm{HMI}$ & $\begin{array}{l}\text { To send the alarming signals by } \\
\text { the users from the PLC program } \\
\text { to HMI, then the HMI displays } \\
\text { I the alarming signals according } \\
\text { to the alarm information } \\
\text { compiled and downloaded to [6] } \\
\text { the control system }\end{array}$ \\
\hline 4 & V16002000 & $\mathrm{HMI} \rightarrow \mathrm{PLC}$ & $\begin{array}{l}\text { HMI sends the effective } \\
\text { alarming response to PLC, such } \\
\text { as PLC stop,Emergency } \\
\text { stop,insert-banning, etc. }\end{array}$ \\
\hline 5 & $\begin{array}{l}\text { V17000000 } \\
\text { V17000003 }\end{array}$ & $\mathrm{HMI} \rightarrow \mathrm{PLC}$ & $\begin{array}{l}\text { To send the program control } \\
\text { signals chosen by the uses to the } \\
\text { PLC(DRY,Program } \\
\text { Testing,ROV) }\end{array}$ \\
\hline 6 & $\begin{array}{l}\text { V25001000 } \\
\text { V25001012 }\end{array}$ & $\mathrm{NCK} \rightarrow \mathrm{PLC}$ & $\begin{array}{l}\text { To send the M signal decoding } \\
\text { by the NC the to the PLC, } \\
\text { including M0 M99 }\end{array}$ \\
\hline 7 & $\begin{array}{l}\text { V30000000 } \\
\text { V30000002 }\end{array}$ & $\mathrm{PLC} \rightarrow \mathrm{NCK}$ & $\begin{array}{l}\text { To return the effective signals } \\
\text { back to the NCK confirmed by } \\
\text { K PLC, including AUTO, JOG, } \\
\text { MDA, back-to-zero control } \\
\text { method and pulse increment }\end{array}$ \\
\hline 8 & $\begin{array}{l}\text { V31000000 } \\
\text { V31000001 }\end{array}$ & $\mathrm{NCK} \rightarrow \mathrm{PLC}$ & $\begin{array}{l}\text { To return the effective signals } \\
\text { back to the PLC confirmed by } \\
\text { NCK }\end{array}$ \\
\hline 9 & V380x0002.1 & $\mathrm{PLC} \rightarrow \mathrm{NCK}$ & $\begin{array}{l}\text { Control the loop through the } \\
\text { coordinate axis tomake it in } \\
\text { control position }\end{array}$ \\
\hline
\end{tabular}

The Assignment Of the Signal Address. Machine external input and output signals include MCP (machine control panel) and machine electrical control signal. The MCP control signals include axis motion control signal, $\mathrm{NC}$ control signals and signal status indicators and so on. These parts of the signals can be connected to the PLC via the connector X111 or X222 of PP72/48. The main electrical control signals of machine include each axis limit signal, motor off-and-on control and overload start signals, electrolysis power supply voltage and current detection signals. This portion of the signal can be connected to the PLC via the connector X333 of PP72/48. PLC embedded in the system contacts the interface variables $\mathrm{V}$ and the corresponding data bits form to control and transmit the information with NCK, HMI and MCP [5].And in accordance with the work state of the system and the control procedure programmed by the users, the logic control tasks of the machine tool control program is completed. The internal signal transmission address and contents of PLC, NCK, HMI, and MCP are as shown in Table I.

\section{System Debugging}

System Initialization. When the System is electrified, the first step is to carry on the system initialization. The machine tool has no spindle, so the initialization of the file "milling machine" hould be chosen according to the type of system and the process requirements. Through the establishment of communication links by RCS802 or through CF card to copy the initialization file $802 \mathrm{ds} 1$ Pro to 802 DSL system. First is the outage of the NC power, and then the initialization file will be took effect after power.

PCL Debugging. After the 802 parts of DSL are sure to connect well, first of all the PLC control logic should be designed and debugged. But with not start to debug drivers and tune 802 DSL parameters until all the safety function of PLC are accurate.

By making use of the "direct network" to connect the computer with 802 DSL X5, and then to start the PLC programming tool and to come into the communication frame in which the Ethernet parameters can be set and the communication links can be established. We download the PLC program edited correctly by using software programming tools to 802Dsl then restart the PLC application. And it is available to use "program status" function to monitor the state of the ladder diagram (not including $\mathrm{L}$ variable). Also, by the way of "map status" function to monitor the state ofthe internal address [7].

When the debugging stop processing subroutine, setting the demand MD14512[16]Bit0=1, it shows the mode of system debugging. The "stop" alarm can be removed by restoration. After the debugging, the parameters can be changed into MD14512[16]Bit0 to "0".The PLC program is based on a subroutine library. The parameters of the tool should be set under the manufacturer's level to PLC MD31060=2, then to define the type ofmachine for" milling machine".

The Debugging of the Drive Control .After the debugging of PLC, the drive debugging can be available. The steps are as follows: 
(1) Loading SINAMICS Firmware aims to ensure that the drive components have the same firmware version;

(2) Loading drive to factory settings aims to activate all parts offactory parameters;

(3) The identification and confirmation of the topology aim to read the connection topology structure of the drive and the control parameters of the motor, then the topology's level is set. When the power of the system and the drive is broken, the modification is not effective until electricity supply again.

\section{The Debugging Of NC.}

(1) Bus configuration. PROFIBUS is defined by the Parameters MD11240 as I/O modules in PP72/48 address, the system is provided with only a PP72/48 addressed ninth.

(2) Positioning drive module. As the machine spindle is not configured, it must be removed from the $\mathrm{NC}$ which parameters are set with MD20070 [3] =0. And through MD20070 to set axis No $\mathrm{X} / \mathrm{Y} / \mathrm{Z} / \mathrm{A}$ with the orderly numbered 1/2/3/4.Through the definition of machine data MD30110 (the axis number of drive shaft port) and MD30220 (the feedback port number of shaft position), $\mathrm{NC}$ axis can be rated corresponding to the correct SINAMICS drive system. And the actual value is feedback from the drive system. The value defined is shown in Table II

TABLE II. AXIS NUMBER DEFINITION

\begin{tabular}{|l|c|c|c|c|}
\hline The axis & $\mathrm{X}$ & $\mathrm{Y}$ & $\mathrm{Z}$ & $\mathrm{A}$ \\
\hline MD30110 & 2 & 3 & 4 & 1 \\
\hline MD30220 & 2 & 3 & 4 & 1 \\
\hline SINAMICS number & 4 & 5 & 6 & 3 \\
\hline
\end{tabular}

(3) The function of the position control. Each axis is set for simulation axis in $t$ he systems factory settings, and then the system will neither read the motor position signal, nor generate instructions to the drivers [8]. The parameter MD30130 (controlling the given type of output) is set to "1", MD30240 (feedback class Type ofthe encoder) is set to "4". Respectively, it shows that NC signals get through the signals of the Profibus output and read the actual value of encoder, which can activate the axis position controller. And the reverse of the motor is adjusted by the parameter MD32100.

(4) The settings ofmachine parameter. 802Dsl defaults the type of X / Y / Z axis as feed shaft, and the machine parameters which need to be set are: MD31030 (the Screw Distance), MD31050 (the gear teeth of the motor side), MD31060 (the gear teeth of screw terminal), MD32000 (the maximum axis Speed). According to the habit of the electrochemical machining operations, Z-axis feed downward is set as "positive", and the entire motor rotation direction can be adjusted by modifying the parameters MD32100. To be able to return to the reference point correctly, the parameters MD34010 (the returning direction of the reference point) is set to "0", and search the reference point of Z-axis forward. The MD34050 (the direction of zero pulse search) is set to "1", and search the Zero pulse at the negative direction. As for the rotational axis A, the parameter MD30300 (IS_ROT_AX) was changed to "1", and it is defined as the spin axis. The parameters MD30310 (drive shaft module) is set to "1" without feed restriction in both the positive and negative direction. The parameters MD30320 (the display mode of Rotary axis coordinate) is set to " 0 ", which means that the rotation axis shows by 360 degrees MODULO axis. If set to " 1 ", it indicates the absolute position with the value of " 0 ". The not-turning number ofthe A shaft encoder pulse is 36000 according to the definition the parameter MD3 1020.

If there is an error or a gap in the screw nut drive, the dial indicator can measure the gap of feed axis in reverse direction, and the error can be compensated up by the parameter MD32450. 63 compensation points can be inserted into the Z-axis via the parameters MD38000, and the laser interferometer can measure each error value and write to the compensation program, which then uploaded to the system processing program.

After the debugging is completed, the data of the machine tool and system procedure can be saved and back-upped.

\section{CONCLUSION}

Based on the analysis of the machine structure and control requirements, we have successfully applied 802Dsl system to the ECM tool. The machining experiments show that the 802Dsl CNC system is security, stability and easy to operate. And its functions of linkage and interpolation can make ECM complete machining ofthe complex parts with simple cathode. The 802Dsl CNC solves the correspondence relationship between the cathode and the workpiece, reduces costs of the cathode's design and manufacture, improves the applicability of ECM, and gives full play to the advantages of ECM. The $\mathrm{CNC}$ system provides a reference for the design of control system of the subsequent ECM tool.

\section{ACKNOWLEDGMENT}

Fund Project: Through the local skill high level university construction project fund support (Project Grant No.: 2015gx013-1-5).

Author: Si-Guo Yang (1963-), male, research directions for the technology and equipment of non-traditional machining, master of engineering, associate professor. E-mail:2849865748@qq.com.Tel.:13705503205

\section{REFERENCES}

[1] Chen Yuanlong,Jia Zhihua. The development of DJK3225 horizontal electrolysis machine tools based on the PLC[J]. The electric processing and die, 2007(6):15-17.

[2] Chen Bingsen,Hu Huali. The PLC programming and application of Siemens $802 \mathrm{D} \mathrm{CNC}$ system[J]. The equipment manufacturing and technology, 2008.9: 148-15 1.

[3] Chen Yuanlong,Li Ming,Tang Huohong. The design of electrochemical deburring machine control system[J].Modular machine Tool \& Automatic Manufacturing Technique,2012. 10(10)63-68

[4] Feng Shihui. The application of PLC control technology in electrochemical machining device $[\mathrm{J}]$. The aerospace manufacturing technology, 2005.6(3):43-46. 
[5] Liu Bin.The application of Siemens 802Dsl in upgrading the crankshaft eccentric grinder $\mathrm{CNC}$ system[J]. Manufacturing Technology \& Machine Tool, 2013 (3): 134-140.

[6] Zheng Shifu, Xu Zhaolin. The application of Siemens 802Dsl in the transformation of Siemens electrical system of $\mathrm{CNC}$ lathe[J].The machine tool and hydraulics,2012. 1 1(22):95-98.

[7] Xu Jian, An Yi.The application of Siemens CNC system in five axis linkage technology transformation[J]. Manufacturing Technology \& Machine Tool,2012 (9): 1 17-120.

[8] Gong Yunhuan,Yi Jian. The application of Siemens 802Dsl in the transformation of the processing center[J].The journal of Sichuan ordnance, 2012.9(9):92-94. 